\title{
La rétention des demandeurs d'asile dans la forteresse britannique (partie 1)
}

Barbara Harrell-Bond et Enoch Opondo

\section{OpenEdition \\ Journals}

\section{Édition électronique}

URL : http://journals.openedition.org/conflits/339

DOI : $10.4000 /$ conflits.339

ISSN : $1777-5345$

Éditeur :

CCLS - Centre d'études sur les conflits lilberté et sécurité, L'Harmattan

Édition imprimée

Date de publication : 15 octobre 1996

ISSN : 1157-996X

\section{Référence électronique}

Barbara Harrell-Bond et Enoch Opondo, «La rétention des demandeurs d'asile dans la forteresse britannique (partie 1) », Cultures \& Conflits [En ligne], 23 | automne 1996, mis en ligne le 15 mars 2006, consulté le 30 mars 2021. URL : http://journals.openedition.org/conflits/339; DOI : https://doi.org/ 10.4000/conflits.339

Ce document a été généré automatiquement le 30 mars 2021.

Creative Commons License 


\title{
La rétention des demandeurs d'asile dans la forteresse britannique (partie 1)
}

\author{
Barbara Harrell-Bond et Enoch Opondo
}

\section{LA RETENTION DES DEMANDEURS D'ASILE DANS LA FORTERESSE BRITANNIQUE}

Enoch OPONDO, Barbara HARRELL-BOND ${ }^{1}$

L'ancien est en train de mourir et le nouveau ne peut pas naître - durant cet interrègne, une grande diversité de symptômes morbides surgit

Antonio Gramsci, Carnets de prison.

En Grande-Bretagne, le droit d'arrêter une personne dont disposent le Ministère de l'Intérieur et le Service de l'Immigration, en vertu de la loi sur l'immigration de 1971 (Immigration Act 1971) affecte directement plus de 10000 personnes chaque année. La plupart des gens arrêtés cherchent à entrer sur le territoire du Royaume Uni et sont détenus dans un lieu proche de l'aéroport pendant que les autorités examinent leurs demandes d'entrée, ou préparent leurs renvois. La durée de la rétention varie de quelques heures à quelques jours pour un voyageur ou une voyageuse qui n'est pas autorisé à passer ses vacances au Royaume-Uni, à plusieurs mois pour les personnes demandant l'asile, parce qu'elles risquent d'être arrêtées, emprisonnées, torturées dans leur pays d'origine. D'autres personnes, ayant vécu avec leurs familles et leurs enfants, parfois plusieurs années, au Royaume-Uni, disposant d'un travail, sont à présent également soupçonnées d'avoir enfreint les lois sur l'immigration et placées en rétention; leur combat pour obtenir l'autorisation de rester au Royaume-Uni avec femmes et enfants peut durer des mois, voire des années. Il n'existe aucune limite de temps obligeant le Ministère de l'Intérieur à prendre une décision et donc aucun moyen de connaître la durée cette épreuve ${ }^{2}$.

6 Cet article examine les conditions de rétention des demandeurs d'asile au Royaume Uni en les resituant dans le contexte du renforcement général des contrôles de l'immigration influencé par les politiques restrictives menées en matière 
d'immigration en Europe, et souvent qualifiée par euphémisme "d'Europe forteresse". Il existe en moyenne 600 demandeurs d'asile détenus au Royaume-Uni qui attendent qu'une décision les concernant soit prise ${ }^{3}$. Bien que ce chiffre puisse paraître peu élevé, il ne doit masquer ni l'angoisse de ceux qui demandent l'asile à la Grande Bretagne, ni la dynamique des politiques d'admission et de refus du Royaume-Uni, ni la perversion des principes de justice, ainsi que le détournement des dispositions du droit international.

7 La première partie de notre article s'attache à définir le contexte historique de la rétention des demandeurs d'asile au Royaume-Uni. La rétention des demandeurs d'asile en Grande-Bretagne s'inscrit en effet dans la tradition de l'internement des étrangers au XXe siècle, même si pour expliquer en partie le recours intensif récent à cette pratique, on présente maintenant cette mesure comme une réaction nationale aux forces qui tentent d'éloigner la Grande Bretagne de sa position traditionnelle d'autonomie à l'égard de l'Europe continentale et on en fait le résultat d'une européanisation dénaturant les traditions britanniques. La deuxième partie s'attache à montrer que les autorités politiques et administratives britanniques ont découvert la puissance de la rhétorique anti-étrangers. Bien qu'elles soient incapables d'empêcher les nationaux des autres pays européens de venir en Grande Bretagne, elles se sont focalisées sur les plus vulnérables, les demandeurs d'asile, cible de la pratique antiétranger d'internement. Nous examinerons dans la troisième partie la légitimité de ces internements pratiqués à l'égard des demandeurs d'asile par la Grande-Bretagne aux regards des textes officiels internationaux dont la Grande Bretagne est signataire, les conditions de rétention, en prenant en compte la durée de rétention des demandeurs d'asile.

Renvoyer les étrangers et les enfermer : pratique ancienne ou récente ?

9 La rétention des demandeurs d'asile en Grande-Bretagne est régie par la loi sur l'immigration de 1971 (Immigration Act 1971), les dispositions générales de la loi sur la nationalité de 1981 (British nationality Act 1981), la loi sur l'immigration de 1988 (Immigration Act 1988) et la loi sur les recours relatifs à l'asile et à l'immigration (Asylum and Immigration Appeals Act 1993). Il existe également les Règlements sur l'immigration (Immigrations Rules), que le Ministère de l'Intérieur a introduits dans la section 3(2) de la loi sur l'immigration de $1971^{4}$. Ces instruments légaux ont été mis en oeuvre au fil des années et forment actuellement le système de contrôle de l'immigration visant à limiter le nombre et la nature de la population étrangère en Grande-Bretagne ${ }^{5}$. L'internement ou la rétention des demandeurs d'asile relève de ce système légal. Ce système est-il ancien et national ou récent et européen ?

10 Perspective historique nationale

11 Même si tout au long du XIXe siècle, la Grande-Bretagne constitua un refuge pour les exilés fuyant la répression ${ }^{6}$, les étrangers étaient déjà perçus comme constituant une présence menaçante à l'époque victorienne. Le déclin économique et la crainte de la concurrence d'une main-d'oeuvre étrangère bon marché dressaient une partie importante de l'opinion publique britannique contre l'immigration sans restriction ${ }^{7}$.

L'une des premières mesures radicales de contrôle de l'immigration fut prise en réponse au grand nombre de Noirs "sans ressources", et de lascars ${ }^{8}$. Le traité commercial de 1813 avec les Indes Orientales (East indian Trade Act of 1813) chercha à 
éviter à l'Etat d'avoir à secourir les étrangers indigents, particulièrement les "marins asiatiques". Ces dispositions furent reprises dans un traité de $1823^{9}$.

Au milieu de l'époque victorienne, le besoin de mettre à l'écart l' étranger imprègne la pensée sociale britannique. Cette attitude fut renforcée par les théories sociobiologiques, comme l'eugénisme, théorie très répandue dès le début des années 1860. Dans les années 1890 et 1900, les sentiments en faveur du droit d'asile ou des étrangers, qui avaient été un trait caractéristique, quoique paradoxal, de la société du milieu des années victoriennes, furent remplacés par une demande de contrôle de l'immigration. Les hommes politiques découvrirent alors la puissance de la rhétorique et de la pratique anti-étrangers comme argument électoral ${ }^{10}$.

14 La première campagne importante contre de nouveaux arrivants intervint à la fin du XIXe siècle avec la venue de réfugiés juifs fuyant la persécution tsariste. Leur arrivée coïncida avec le déclin économique britannique. La campagne en faveur d'une loi sur les étrangers reçut une impulsion déterminante des quartiers Est de Londres où nombre de réfugiés s'établissaient, mais où les Anglais de naissance les percevaient comme une menace économique et sociale ${ }^{11}$.

La loi sur les Etrangers de 1905 (Aliens Act), adoptée après deux décennies d'agitation intermittente, constitue à cet égard un point de référence dans le déclin du libéralisme. Elle déclencha une érosion irréversible de la notion d'asile comme idéal et comme pratique. La Loi conféra aux Officiers de l'Immigration le pouvoir de refuser l'entrée aux personnes considérées comme « indésirables ». Le Ministère de l'Intérieur obtint le pouvoir d'expulsion sur avis du tribunal. Cette loi marqua une évolution majeure de la pratique antérieure selon laquelle l'entrée des personnes qui n'étaient pas des sujets britanniques relevait techniquement de la seule prérogative royale, le monarque pouvant aussi faire expulser des individus ou des groupes de personnes ${ }^{12}$.

16 La prolifération législative qui suivit la loi de 1905 visa à renforcer le contrôle de l'immigration par le développement et la mise en oeuvre des principes de base contenus dans la Loi de 1905. Des pouvoirs de contrôles accrus sur l'entrée et la sortie des étrangers furent attribués au Ministère de l'Intérieur, avec l'obligation pour les étrangers d'être enregistrés, notamment par la police ${ }^{13}$.

17 La dynamique du processus se fonda sur la relation existant entre les Britanniques, les hommes politiques et le Ministère de l'Intérieur, chaque élément radicalisant l'autre. Le public apprit que les vociférations, pour localisées et limitées qu'elles fussent, pouvaient réussir à fermer la porte aux réfugiés et autres migrants. Les hommes politiques s'aperçurent qu'il y avait beaucoup à gagner et peu à perdre à flatter l'hostilité aux étrangers. Les responsables du Ministère de l'Intérieur acquirent une expérience dans l'organisation du contrôle des entrées sur le territoire ainsi que dans la gestion des étrangers à l'intérieur du pays ${ }^{14}$.

18 L'hostilité à l'égard des étrangers fut renforcée par les années de guerre et persista durant la période de paix, accrue par les nouvelles peurs comme la menace de la révolution, la drogue, la peur de la dégénérescence. Le discours contre les étrangers pris l'allure d'une toile d'araignée sans faille. Les mêmes stéréotypes sur l'étranger « autre » furent utilisés pour les Juifs, les Allemands, les Noirs, les Chinois et les Gitans. Les inquiétudes sur l'école, la santé publique, les codes moraux, et par dessus tout, l'identité nationale, prirent pour cible les immigrants et les minorités. La réponse à l'immigration des réfugiés Juifs et la vague d'internement de 1940 furent en conséquence à peine atypiques. Elle était compatible et cohérente, avec le cadre 
constitué par les attitudes populaires, les comportements politiques et les pratiques officielles ${ }^{15}$.

19 Avec l'élaboration du décret sur l'immigration du Commonwealth de 1962 (Commonwealth Immigration Act) qui fut adopté sur fond d'agitation et de pression racistes, s'affirma une tendance raciste et anti-noire. Cette loi et les suivantes, adoptées en 1968 et 1971, aboutirent à un parti pris raciste, attendu qu'elles «étaient primitivement conçues pour empêcher l'entrée des sujets britanniques noirs (citoyens du Commonwealth et du Royaume-Uni); d'où l'invention du "droit de résidence" (initialement appelé "patrialité") et du traitement préférentiel appliqué aux citoyens du Commonwealth dont un grand parent était né au Royaume-Uni, afin de s'assurer que nombre d'Australiens, de Néo-zélandais et de Canadiens ne soient pas exclus par la législation ${ }^{16}$.

Ces lois entraînèrent l'exclusion de Grande-Bretagne de certains nationaux britanniques (presque tous noirs), alors que certains nationaux (pour la plupart blancs), provenant des pays du Commonwealth furent libres d'entrer à tout moment. L'entrée de la Grande-Bretagne dans la Communauté Economique Européenne le 1er janvier 1973 (date qui coïncida avec l'entrée en vigueur de la loi sur l'immigration de 1971) apporta de nouveaux droits à l'entrée et au travail à des centaines de millions de nationaux européens. Cette loi et la loi sur la nationalité britannique de 1981 (British Nationality Act 1981) ont codifié en Grande-Bretagne la législation sur l'immigration et la nationalité. Depuis lors les nouvelles lois, les règlements et les pratiques ont imposé des restrictions croissantes aux personnes entrant dans le pays, particulièrement pour celles venant du Sud. La pratique du Ministère de l'Intérieur à l'égard des demandeurs d'asile devint encore plus restrictive avec la loi sur les recours relatifs à l'asile et à l'immigration de 1993 (Asylum and Immigration Appeals Act 1993) et les nouvelles règles. Cela entraîna une extension de la pratique de la rétention des demandeurs d'asile et la perte de leurs droits de recours ${ }^{17}$. La loi de 1993 - il faut le souligner - porte la marque des décisions européennes intergouvernementales. Entrée en vigueur pendant l'été 1993, elle abolit le droit de recours pour les "visiteurs" qui se sont vus refuser l'entrée sur le territoire. Ceci ne fit que renforcer les pouvoirs déjà considérables des responsables du Service de l'Immigration du Royaume-Uni pour entrer au Royaume-Uni.

21 Le contexte européen et la modification de l'ordre international

22 Dans les années d'après-guerre, caractérisées par le déclin de la Grande-Bretagne sur le plan international et, par la peur morbide d'une guerre "réelle" éclatant entre les deux blocs idéologiques, l'influence de l'Europe continentale sur l'élaboration de la politique britannique a augmenté progressivement. Elle s'est manifestée aux niveaux des actes et des procédures, notamment dans le domaine des migrations au moment où l'Europe élargit ses frontières. Quelques-uns des récents développements européens qui constituent la toile de fond de la réflexion sur la question des demandeurs d'asile en Grande-Bretagne et fournissent un éclairage sur les développements ultérieurs, peuvent être résumés comme suit.

On a assisté à la multiplication d'accords intergouvernementaux sur les différentes questions relatives à l'immigration, dont beaucoup affectent directement ou indirectement les demandeurs d'asile ou les réfugiés. Ils concernent le processus d'harmonisation européenne avec l'Acte Unique, le Traité de Maastricht sur l'Union Européenne et la coopération croissante entre les ministres et les fonctionnaires des 
différents pays européens ${ }^{18}$. Citons l'accord de Schengen sur l'abolition graduelle des contrôles aux frontières entre un nombre croissant de pays du continent; la Convention de Dublin et les projets de conventions parallèles sur le pays approprié pour déterminer l'application du droit d'asile, et les projets de conventions sur le franchissement des frontières extérieures. Il existe une pression, au niveau communautaire, pour favoriser l'abolition des contrôles aux frontières intérieures. Il existe donc une tendance à soustraire les consultations intergouvernementales d'un vote du public sur toutes ces questions et sur d'autres ; en outre, le Traité de Maastricht sur l'Union Européenne "poursuit l'européanisation de la politique d'asile d'une manière typiquement non démocratique ». Enfin il y a de fortes pressions européennes pour l'harmonisation des lois sur l'asile ${ }^{19}$. Dans cette configuration émergente, l'asile est perçu par les Etats européens comme un lien faible dans un système vigoureusement attaché à la notion de frontière.Par ailleurs, s'ajoutant aux mesures déjà prises, l'Union Européenne s'apprête à élaborer, en 1996, une liste commune de visas aussi restrictive que celle de Schengen.

Il apparait en conséquence que la politique du Royaume-Uni à l'égard des demandeurs d'asile comporte deux dimensions: d'une part l'hostilité post-victorienne envers les étrangers, incitant à des efforts permanents pour renforcer les conditions dans lesquelles les étrangers seront autorisés à vivre en Grande-Bretagne, continue ; d'autre part l'impossibilité pour la police de contrôler les entrées de citoyens d'autres Etats européens en Grande-Bretagne, fait se retourner contre les pays du Sud l'hostilité antiétranger. Avec une bureaucratie qui s'est développée au fil des ans pour renforcer les contrôles sur l'immigration, et des hommes politiques qui ne répugnent pas à tenir des discours hostiles aux étrangers pour s'assurer des gains politiques, on peut dire que le demandeur d'asile apparaît comme le point de convergence de toutes les forces de contrôle d'immigration britannique. Leur détention en est une manifestation dont la gravité (au regard de la violation du droit international) ne peut être plaidée pour justifier le "refoulement " des demandeurs d'asile.

Nature de la détention des demandeurs d'asile en Grande-Bretagne depuis 1989

«Des fonctionnaires inconnus détiennent apparemment maintenant des pouvoirs despotiques considérables très difficiles à contester $"{ }^{20}$.

Les demandeurs d'asile détenus, en vertu de la loi sur l'immigration de 1971, sont placés dans les centres de rétention pour immigrés, dans les prisons pour criminels ou dans les cellules des postes de police. La Loi sur l'Immigration de 1971 donne le droit à la police et aux services de l'immigration d'arrêter les personnes sans mandat. Dans les cellules de la police et dans les prisons de Sa Majesté, les détenus sont traités comme des prisonniers en détention provisoire. Ils peuvent être enfermés dans des petites cellules, privés de moment de détente ou d'exercice vingt heures sur vingt-quatre. La différence majeure qui existe entre les criminels de droits commun et ces détenus est que ces derniers peuvent être retenus indéfiniment sans jugement. En 1989, le service d'immigration définissait sa ligne de conduite en matière de rétention comme suit: «détenir seulement lorsqu'il est essentiel d'agir ainsi et n'accorder l'admission temporaire que lorsque le fonctionnaire d'immigration a la certitude que l'individu se conformera à toutes les restrictions imposées ».

Selon les instructions du service d'immigration en effet la rétention ne doit être utilisée qu'en dernier ressort. Elle ne devrait intervenir qu'à la fin du processus, une fois épuisées tous les possibilités de recours... Les éléments qui motivent le maintien en 
rétention d'un demandeur d'asile, lorsque celui-ci fait valoir ses droits, doivent être particulièrement probants ${ }^{21}$. Seulement, est-ce toujours le cas ? Pourquoi toujours plus de demandeurs sont-ils détenus?

Le Ministère de l'Intérieur, dans les années qui suivirent cette déclaration, augmenta en fait ses capacités de rétention. Le 29 novembre 1993, le centre d'immigration de Campsfield House (situé près de l'aéroport d'Oxford, à environ six miles au Nord de la ville ) fut ouvert. Les services du ministère annoncèrent plus tard que trois nouveaux centres de rétention seraient construits en 1995 près des aéroports de Gatwick, Heathrow et Stansted. Pour faire face au nombre croissant de demandeurs d'asile détenus dans les prisons et les centres de rétention pendant des périodes prolongées (c'est-à-dire plus d'un an), pendant l'instruction de leurs dossiers, le Ministère de l'Intérieur lança une seconde phase de développement des capacités d'accueil des détenus. Le 13 Juin 1994, on annonça que pas moins de 500 demandeurs d'asile et immigrants seraient détenus dans des ailes spéciales de cinq prisons. Birmingham, Rochester dans le Kent, Holloway à Londres, Doncaster et Hasler Holding Centre à Gosport (Hampshire). Ces prisons devaient remplacer les 41 lieux vétustes qui avaient été utilisés jusqu'à cette date.

Cette tendance à accroître les capacités de rétention en utilisant des prisons fut critiquée, car elle allait à l'encontre du principe juridique fondamental de présomption d'innocence avant que ne soit apportée la preuve de la culpabilité. L'inspecteur principal des prisons, quant à lui, avait fait valoir dès 1994, que l'utilisation de prisons d'époque victorienne pour la rétention des demandeurs d'asile conduisait ces derniers au désespoir et à des actes d'auto-mutilation ${ }^{22}$. La concentration des moyens sur cinq prisons fut la réponse du service des prisons à cette critique. La thèse officielle présentait la concentration des moyens comme permettant au service des prisons et au département ministériel responsables des problèmes d'immigration et de nationalité de résoudre plus facilement les questions pratiques liées à la langue, à la traduction et à l'assistance. Cette réponse n'abordait pas en revanche la question fondamentale de l'emprisonnement de personnes innocentes.

Or, les demandeurs d'asile détenus ne sont ni accusés ni reconnus coupables d'un quelconque crime ; ils n'ont comparu devant aucun tribunal. Ils sont détenus en vertu des pouvoirs administratifs dont dispose le Ministère de l'Intérieur ou le service de l'immigration et, ne peuvent pas généralement demander une mise en liberté sous caution. Dans la mesure où leur rétention est administrative, la juridiction normale du tribunal ne s'applique pas et le Ministère de l'Intérieur n'a pas à justifier la rétention devant une assemblée publique. Peu de détenus sont clairement informés des raisons spécifiques de leur rétention et, à la différence des prisonniers criminels en détention provisoire, nombre d'entre eux n'ont pas le droit de demander une mise en liberté sous caution. En outre, même ceux qui peuvent y prétendre sont vraisemblablement dans l'impossibilité de le faire, ne serait-ce que parce qu'ils ne peuvent pas donner les 4.000 $£$ de garantie qui sont exigées ${ }^{23}$. Il n'y a pas de limite à la durée de la rétention administrative des demandeurs d'asile et aucun organisme de contrôle ne surveille la manière dont se passe la rétention. Ceci crée une situation où les demandeurs d'asile sont détenus pendant des périodes prolongées sans accusation retenue contre eux, sans avoir été jugés, condamnés. Les personnes détenues ne disposent d'aucun droit légal de connaître les raisons de leur rétention. La seule raison avancée par les officiers d'immigration pour justifier cette situation est que si l'on accorde l'admission 
temporaire aux personnes détenues, elles ne respecteront pas l'échéance fixée. En d'autres termes, elles sont maintenues en rétention sur simple soupçon. La prolongation de la rétention est réexaminée, non par les magistrats ou par un organisme impartial, mais par les fonctionnaires du Ministère de l'Intérieur. vereée. Elles peuvent se réduire à un entretien plus détaillé, éventuellement avec le concours d'un interprète du Ministère de l'Intérieur. Dans d'autres cas, elles comportent un long interrogatoire sur les visites antérieures effectuées au RoyaumeUni en s'appuyant sur des renseignements fournis par une interrogation de données informatisées. Si le fonctionnaire de l'Immigration n'est pas satisfait, il rédige une note officielle motivant le refus d'admission. Ce fonctionnaire a alors la possibilité de retenir le passager jusqu'à son renvoi, et le pouvoir discrétionnaire d'accorder une admission temporaire. Pour un demandeur d'asile, l'officier d'Immigration conduit un premier entretien réglementaire destiné à établir s'il est passé dans un autre pays avant d'arriver au Royaume Uni et s'il est raisonnable de l'y (ou de la) renvoyer pour y solliciter l'asile. Dans le jargon de l'immigration, cela s'appelle "un renvoi vers un pays tiers sûr". Si la personne est bien passée par un autre pays, le compte-rendu de cet entretien est faxé au Ministère d'Intérieur. Quelques heures plus tard, le Ministère de l'Intérieur faxe une réponse aux officiers d'Immigration du port, pour autoriser l'éloignement vers le pays tiers. Ils examinent alors s'il existe des motifs selon les règles de l'immigration justifiant d'accorder l'entrée. Si aucun motif n'est trouvé, un avis de refus d'admission et des dispositions de renvoi vers le "pays tiers sûr" sont remis aux passagers. Les fonctionnaires de l'Immigration prennent habituellement des dispositions rapides de renvoi tout en retenant entre-temps les passagers. Selon un accord intervenu entre le Ministère de l'Intérieur et le Centre officiel des Réfugiés, tous les cas de cette nature doivent être soumis à ce Centre. Ce dernier dispose d'un délai de 48 heures pour présenter les arguments s'opposant au renvoi. Le Ministère de l'Intérieur peut prendre en considération les inconvénients liés à la nature du pays tiers ou à l'existence de membres proches de la famille du demandeur d'asile vivant au Royaume-Uni.

Cultures \& Conflits, 23 | automne 1996 
- Attente d'une décision autorisant ou non le passager à entrer. Le cas intervient le plus souvent lorsque le passagerrevendique immédiatement l'asile au Royaume-Uni. Les officiers de l'Immigration ne sont pas à même de prendre une décision et sont tenus de s'en remettre au département du ministère de l'Intérieur traitant des questions d'asiles et des cas spéciaux. Jusqu'à ce qu'une décision ait été prise, le service de l'immigration ne peut pas apprécier si le passager remplit les conditions pour qu'on le laisse entrer en vertu des dispositions contenues dans les lois sur l'immigration.

39

- Après un refus de laisser entrer un passager et en attendant son éloignement du Royaume-Uni. Ceci se produit lorsque l'Officier de l'Immigration estime que le passager ne répond pas aux exigences du droit d'entrée en vertu des lois sur l'immigration, par exemple parce qu'il a des doutes sur l'authenticité des documents de transport du passager ${ }^{24}$. avons déjà indiqué que la loi n'impose aucune limite de temps à la rétention administrative. Une telle rétention ne peut normalement faire l'objet d'aucune révision, de même que, comme indiqué, les cautions pour mise en liberté provisoire ne peuvent s'appliquer ${ }^{25}$

Si les officiers de l'Immigration décident de retenir le passager, l'officier procédant à l'entretien en réfère au fonctionnaire supérieur responsable de l'Immigration qui autorise la rétention. Les personnes retenues sont alors escortées par des gardes de sécurité privée vers les douanes, où leurs bagages et vêtements sont examinés. Parfois, cet examen entraîne une fouille corporelle complète avec déshabillage qu'on justifie habituellement par la recherche de drogues. Les détenus sont emmenés ensuite dans un centre de rétention, où le personnel d'escorte remet l'ordre de rétention, l'autorisation d'être transféré dans ce centre et le formulaire d'information sur la détention. Le Service de l'Immigration insiste sur le fait qu'il n'est pas tenu de justifier la rétention. Cependant des raisons sont quelquefois avancées. On peut citer, parmi d'autres, les suivantes :

- Fraude (documents falsifiés, tentatives d'obtenir une entrée en tant que visiteur ou ensuite réclamer l'asile etc.);

- Fraude importante (essayer d'entrer avec un titre britannique de voyage); 
44

- Cas d'asile non probant ;

45

- Manque de liens communautaires (ne connaît personne dans le pays et/ou ne dispose d'aucun endroit où aller pour vivre)

46

- manque de ressources ;

47

- Demande de détention pour sa propre sécurité ;

48

- Lorsque la personne est dans l'attente d'une décision rapide sur sa demande d'asile et préfère être retenue jusque là ;

49

- Doute sur l'identité et/ou la nationalité de la personne retenue ;

50

- Si la personne retenue essaye de s'enfuir de l'aéroport.

5

- Lorsque les fonctionnaires de l'Immigration ne sont pas satisfaits par les faits et que la personne demeure dans les conditions de l'admission temporaire.

52

- Lorsque la personne est identifiée "clairement" comme un immigrant économique"26.

Admission temporaire

54 temporaire d'un demandeur d'asile détenu ou susceptible d'être détenu. Ils doivent notifier l'admission temporaire par écrit, peuvent fixer les conditions de résidence et un contrôle au poste de police ou à un bureau d'immigration; ceci empêche le plus souvent l'accès à un emploi. L'admission temporaire n'est pas une permission légale d'entrer dans le pays mais seulement une alternative à la rétention. Le pouvoir d'admission temporaire fut créé par la loi sur l'Immigration de 1971 et est appliquée depuis le 1er janvier 1973. Cependant jusqu'à la fin des années 70, le Service de l'Immigration n'a que peu utilisé ces pouvoirs devant les pressions émanant de différentes organisations au Royaume-Uni. En 1979, le Ministre responsable de l'immigration déclara que l'admission temporaire serait seulement envisagée lorsqu'elle serait estimée "pratiquable et souhaitable". Il ajouta que si on accordait davantage d'admissions temporaires: "il était raisonnable de s'attendre à une augmentation de fuites". En conséquence, des membres du Parlement furent sollicités pour présenter des requêtes en faveur des personnes retenues pour que leur soit accordée l'admission temporaire ${ }^{27}$.

Lire la suite 


\section{NOTES}

1. Barabara Harrell-Bond est Directrice du Refugee Studies Programme, Université d'Oxford. Enoch Opondo est maître de Conférences en science politique à l'Université Moi au Kenya, et Directeur adjoint du Centre for Refugee Studies de l'Université.

2. Ashford M., Detained Without Trial : A Survey of Immigration Act Detention London : JCWI, 1993, p. xi.

3. Amnesty International, Prisoners Without A Voice : Asylum-Seekers Detained in the United Kingdom (Second Revised and Updated Edition), London, Amnesty International British Section, 1995.

4. Warr G., Immigration Handbook, London, Sweet and Maxwell, 1994, p. 3.

5. Le système actuel de contrôle de l'immigration s'est mis en place au début du XXe siècle avec l'adoption de la Loi sur les étrangers de 1905 (Aliens Act 1905), Joint Council for the Welfare of Immigrants, Immigration and Nationality Law Handbook : A User's Guide to the System of British Immigration, Nationality and Refugee Law, London, JCWI, 1995 Edition, p. 4.

6. Panayi P., "Refugees in Twentieth Century Britain : A Brief History » in Robinson V. (ed.), The International Refugee Crisis : British and Canadian Responses London : Macmillan, 1993, p. 95.

7. Cesarani D., kushner T. (eds.), The Internment of Aliens in Twentieth Century Britain, London, Frank and Co., 1993, p. 11. Panayi P., op. cit., p. 95.

8. Nom utilisé pour désigner les marins asiatiques sur les bateaux britanniques, jusqu'à l'indépendance indienne en 1947.

9. Gordon P., Policing Immigration : Britain's Internal Controls, London and Sydney, Pluto Press, 1985, p. 5-6. Deux instruments juridiques, une loi de 1849 et des conventions conclues avec des lascars en 1894 abordaient le problème des lascars. La loi de 1849 considérait ces derniers comme des ressortissants britanniques aussi longtemps qu'ils se trouvaient à bord de navires britanniques, les conventions leurs enjoignaient de retourner dans leur pays d'origine. Les patrons propriétaires de bateaux pouvaient être condamnés au paiement d'une amende si des marins asiatiques ou africains étaient découverts sans ressources ou reconnus coupables de vagabondages dans les 6 mois après leur date de débarquement en Grande-Bretagne. Gordon P., op. cit., p. 107.

10. Cesarani D., kushner T. (eds.), The Internment of Aliens in Twentieth Century Britain, London, Frank and Co., 1993, p. 11.

11. Panayi P.,op.cit., p. 95.

12. Joint Council for the Welfare of Immigrants, op. cit., p. 4.

13. Les lois furent adoptées et amendées en 1914, 1919, 1920, 1925, 1962, 1968, et 1969. Pour un résumé de leurs principales dispositions voir Gordon P., op. cit., p. 107-109.

14. Cesarani D., kushner T. (eds.), op. cit., p. 11-12.

15. Op. cit, p. 12.

16. Joint Council for the Welfare of Immigrants, op. cit., p. 5.

17. Joint Council for the Welfare of Immigrants, op. cit.

18. Op. cit. 
19. Randall C., " An Asylum-Policy for the UK » in Spencer S. (ed.), Strangers and Citizens : A Positive Approach to Migrants and Refugees London, IPPR/Rivers Oram Press, 1994, p. 203.

20. D'après une lettre au rédacteur en chef, The Guardian, 27 Décembre 1993.

21. Voir Campsfield Monitor, $\mathrm{n}^{\circ}$ 6, jan-feb 1995, p. 2.

22. Tumim S., «Immigration Detention Centre - Campsfield House : Report of an Unannounced Short Inspection by HM Inspectorate of Prisons », London, Home Office, 1995.

23. Amnesty International, op. cit., p. 1.

24. Ashford M., Detained Without Trial : A Survey of Immigration Act Detention London : JCWI, 1993, p. 1.

25. Op. cit, p. 1-2.

26. Op. cit, p. 6.

27. Cette clause conditionnelle a depuis été abrogée par la Loi de 1993 sur les recours relatifs à l'asile et à l'immigration.

INDEX

Index géographique : Royaume Uni

Mots-clés : centre de rétention, demandeurs d'asile 